\title{
Acute pneumonitis or vasculitis? A severe case with multiple organ involvement
}

\author{
Laura Balzarini, ${ }^{1}$ Chiara Mancini, ${ }^{1}$ Mario Uccelli, ${ }^{2}$ Enrico Betri, ${ }^{3}$ Maurizio Marvisi ${ }^{4}$ \\ ${ }^{1}$ Internal Medicine, Casa di Cura Figlie di S. Camillo, Cremona; ${ }^{2}$ Division of Radiology, Casa di Cura Figlio di San Camillo, \\ Cremona; ${ }^{3}$ Department of Pathology, Istituti Ospitalieri di Cremona, Cremona; ${ }^{4}$ Department of Medicine, Casa di Cura Figlie \\ di S. Camillo, Cremona, Italy
}

\begin{abstract}
This report describes a case of a 37-year-old man affected by weakness, cough, fever and arthralgia for three months and a single episode of arthritis affecting the left ankle. The symptoms worsened and he was admitted to our hospital. First level blood tests showed an increase in inflammatory proteins, leukocytosis with eosinophilia, positive anti-neutrophil cytoplasm antibody (ANCA) and PR-3 ANCA antibodies, initial renal failure with elevation of creatinine and microscopic hematuria and initial proteinuria in the urine tests. As the chest x-ray revealed a perihilar pneumonitis with a well-defined margin area in the right lung, we started antibiotic therapy. The lung was studied with high-resolution chest computed tomography, which showed interstitial lung disease with more consolidative areas, some of which had a reverse halo sign. As clinical and laboratory data suggested a multiple organ involvement, second level tests were performed to look for the presence of systemic vasculitis.
\end{abstract}

\section{Case Report}

A 37-year-old man was admitted to our unit in June 2014 with a three-month history of fever and arthralgia, and with one transient episode of arthritis affecting the left ankle some weeks before and localized erythema of the same leg, probably erythema nodosum. He had no relevant anamnestic history apart from inhalant allergy.

In March he underwent a specialist evaluation for arthralgia with the prescription of blood tests [rheumatoid factor (RF) was positive, whereas anti-CCP, ANA, ENA, native-dsDNA antibodies and HLA B27 were negative] and of thyroid scintigraphy (never per-

Correspondence: Laura Balzarini, Internal Medicine, Casa di Cura Figlie di S. Camillo, via Fabio Filzi 56, 26100 Cremona, Italy.

Tel.: +39.0372.421111 - Fax: +39.0372.421245.

E-mail: 1r.balzarini@gmail.com

Key words: Vasculitis; granulomatosis with polyangiitis; reverse halo sign.

Received for publication: 8 December 2014.

Revision received: 8 December 2014.

Accepted for publication: 15 December 2014.

This work is licensed under a Creative Commons Attribution NonCommercial 3.0 License (CC BY-NC 3.0)

(C) Copyright L. Balzarini et al., 2015

Licensee PAGEPress, Italy

Italian Journal of Medicine 2015; 9:367-369

doi:10.4081/itjm.2015.572 formed), because echographic signs of thyroiditis and positive thyroid antibodies were found.

Although he did not complain of dyspnea, he had a non-productive cough, tiredness and joint stiffness.

There were right-sided crackles on respiratory examination, while other observations were normal.

Chest $\mathrm{x}$-ray showed perihilar pneumonitis in the right lung with a well-defined margin area.

Laboratory tests revealed an increase in inflammatory markers [C-reactive protein: $15.63 \mathrm{mg} / \mathrm{dL}(>5$ $\mathrm{mg} / \mathrm{dL}$ positive)], moderate leukocytosis with

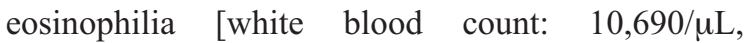
eosinophils $17.76 \%(1900 / \mu \mathrm{L})]$, thrombocytosis [platelets: 507,000 K/ $\mu \mathrm{L}$ ], positive RF [125.39 IU $/ \mathrm{mL}$ (>20 IU/mL, positive)], ferritin $817.4 \mathrm{ng} / \mathrm{mL}(25-300$ $\mathrm{ng} / \mathrm{mL}$ ), mild alteration of renal function tests (serum creatinine: $1.41 \mathrm{mg} / \mathrm{dL})$, proteinuria $(1 \mathrm{~g} / \mathrm{L})$ and microhematuria (erythrocytes $250 / \mathrm{kL}$ ) in the urine test.

We observed positive anti-neutrophil cytoplasm antibody (ANCA) and PR-3 ANCA antibodies (PR-3 ANCA $>8 \mathrm{U} / \mathrm{mL}$ positive and MPO ANCA antibodies were negative).

The patient was treated with large spectrum antibiotics (ampicillin/sulbactam and azithromycin), but we suspected a multisystemic disorder because of the involvement of several organs. As the first symptom was fever and then arthralgia with a transient episode of erythema nodosum, with contemporary involvement of the lung (with cough and later dyspnea) and kidney (with proteinuria and microhematuria), we decided to perform second level tests.

The high-resolution chest computed tomography (CT) showed interstitial lung disease and more consolidative areas, some of which had a reverse halo sign 
(Figure 1). This radiological pattern is defined as a central ground-glass opacity surrounded by denser consolidation and can be observed in other pathologies (Table 1). Bronchoscopy with broncho-alveolar lavage showed a prevalence of erythrocytes as diffuse alveolar hemorrhage.

The presence of PR-3 ANCA antibodies associated with the involvement of the lung and kidney could be indicative of granulomatosis with polyangiitis. To confirm the diagnosis the patient was transferred to the Division of Nephrology, where a kidney biopsy was performed. It showed the presence of segmental necrosis and extracapillary proliferation on the analyzed glomeruli and their fragments (Figure 2).

The patient was treated with intravenous cyclophosphamide (1200 mg; the dosage was reduced to $500 \mathrm{mg}$ at the next cycle) in association with 2-mercaptoethanesulfonate sodium (MESNA) and methylprednisolone (a daily dose of $1 \mathrm{~g}$ for three days running). He was discharged with steroid support (prednisone $60 \mathrm{mg}$ ) and trimethoprim/sulfamethoxazole. The patient had a cutaneous reaction after a few days, probably due to trimethoprim/sulfamethoxazole, which was therefore stopped.

After a transient worsening of creatinine value (1.7 $\mathrm{mg} / \mathrm{dL}$ ), renal function normalized; there was a reduction in inflammatory tests and an improvement of symptoms.

One month later, high-resolution chest CT revealed the resolution of the pulmonary organized areas and the ground-glass opacity.

\section{Discussion and Conclusions}

In 1936 Friedrich Wegener, a German pathologist from Breslau, first recognized this kind of vasculitis, which for decades was named Wegener's granulo-

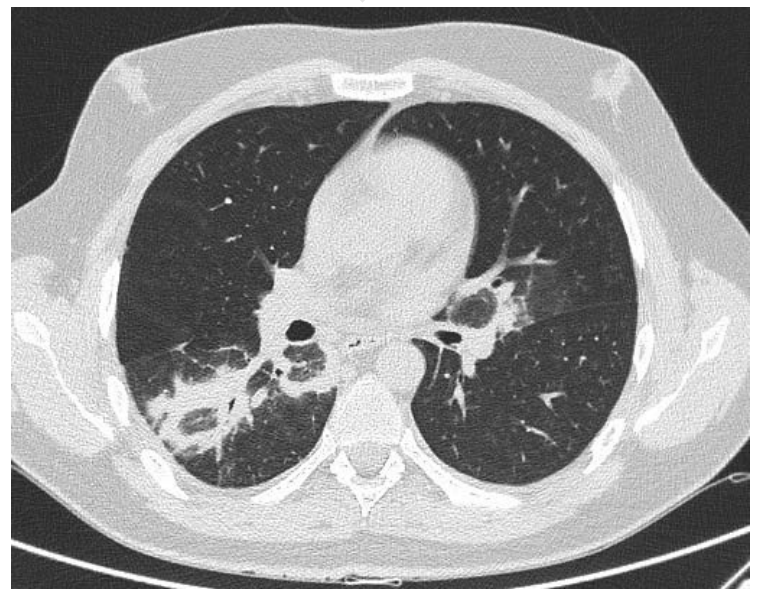

Figure 1. Chest computed tomography shows reverse halo sign in the right lower lobe. matosis. In 2011, the American College of Rheumatology, the American Society of Nephrology and the European League Against Rheumatism recommended a change of name, because of the evidence that Wegener had been a member of the Nazi party before and during World War II. They suggested the name on the basis of pathogenesis and not of the first descriptor of the disease, and proposed the term granulomatosis with polyangiitis (abbreviated to GPA). ${ }^{1}$

GPA is a rare disease, with a close association with ANCA, involving the small and medium-sized blood vessels and damaging tissues with granulomatous inflammation. It often affects the kidney, lung and upper respiratory tract but can affect other organs. The most severe manifestation is glomerulonephritis, which can

Table 1. Reversed halo sign on high-resolution chest computed tomography: differential diagnosis.

GPA (Wegener's granulomatosis)

Cryptogenetic organizing pneumonia

Sarcoidosis

Pneumocystis pneumonia

Opportunistic invasive fungal infection (Paracoccioidomycosis, Histoplasmosis, Cryptococcosis)

Tuberculosis

Community-acquired pneumonia

Pulmonary infarction

Lymphomatoid granulomatosis

Exogenous lipoid pneumonia

Metastasis

Recent radiofrequency ablation of pulmonary malignancies

GPA, granulomatosis with polyangiitis.

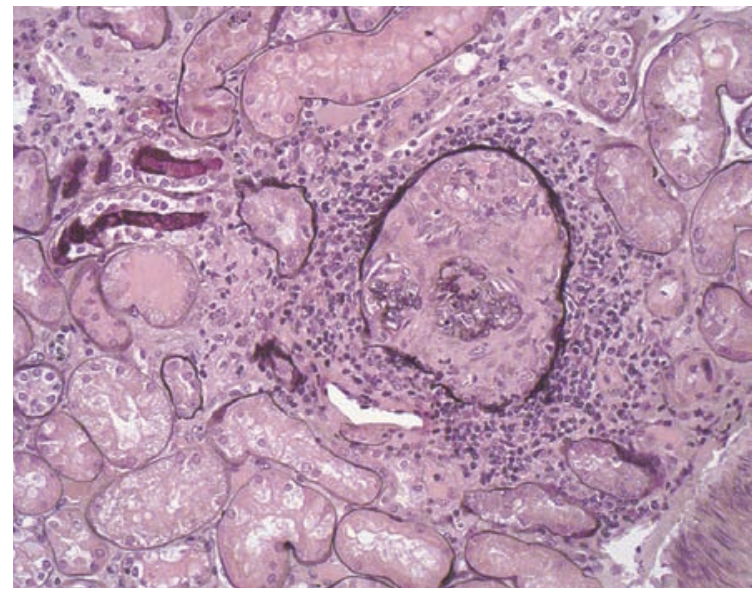

Figure 2. The renal biopsy shows necrosis, extracapillary proliferation and diffuse interstitial leukocyte infiltration. 
evolve into renal failure and alveolar capillaritis, possibly leading to respiratory failure due to lung hemorrhage and permanent organ damage, and it can be fatal if left untreated.

ANCA antibodies in GPA most commonly give a cytoplasmic pattern by immunofluorescence (CANCA anti proteinase 3). The small-vessel vasculitides associated with ANCA are a group of disorders characterized by variable patterns but classified together as ANCA-associated vasculitis (AAV). The other vasculitides of the group, often overlapping each other, are microscopic polyangiitis and eosinophilic granulomatosis with polyangiitis (also known as Churg-Strauss syndrome, the change of name for the same reason as in the case of Wegener).

Our patient was affected by pulmonary-renal syndrome, which is the most common presentation of AAV. Two thirds of AAV are GPA. ${ }^{2}$ The latter is a rare disease, with an estimated prevalence in European countries of 24 to 157 per million, with estimated annual incidence rates of 3 to 14 per million. ${ }^{3}$

AAV causes end-stage renal failure in more than $20 \%$ of patients 5 years on and a 5 -year survival rate of approximately $75 \%$.

A percentage of $85-90 \%$ of treated patients go into remission. ${ }^{4}$ The disease has a relapsing-remitting course, with $50 \%$ relapse rate within 5 years. ${ }^{5}$

The risk of underestimating the symptoms is high, first of all because renal involvement is a silent disease with a tardy diagnosis, when renal damage is significant and irreversible.

There are various symptoms and the diagnosis needs an accurate evaluation of all clinical signs.

Our patient was treated according to the European League Against Rheumatism (EULAR) guidelines, which involve the designing and testing of a regimen of intravenous cyclophosphamide at a dose of 15 $\mathrm{mg} / \mathrm{kg}$ (max $1.2 \mathrm{~g}$ ) every 2 weeks for the first three pulses, followed by infusions every three weeks for the next 3-6 pulses. Patients should also receive oral or intravenous MESNA, which interacts with a toxic metabolite of cyclophosphamide (acrolein), rendering it non-toxic. The immunosuppressive therapy is associated with prednisolone or prednisone at 1 $\mathrm{mg} / \mathrm{kg} /$ day. ${ }^{6}$ Moreover trimethoprim/sulphamethoxazole (800/160 $\mathrm{mg}$ twice daily) is added to standard therapy as preventive care for pneumocystis and also because it seems to reduce the risk of relapse in GPA.

The therapy should be continued for at least 18 months; recent guidelines published by the British So- ciety for Rheumatology recommend therapy for at least 24 months. Early cessation of therapy is associated with an increased risk of relapse. Remission induction treatments in relapsing, refractory or persistent disease consider alternative drugs. For patients who fail to achieve remission and have persistent low activity, intravenous immunoglobulins can be used. For patients with progressive disease in spite of optimal therapy, alternative options include conventional immunosuppressants such as mycophenolate mofetil and 15-deoxyspergualin, and biologic agents such as antithymocyte globulin, infliximab and rituximab. ${ }^{6}$

Our patient had remission with the combination of cyclophosphamide and prednisone.

In this case it was essential to check the involvement of each organ with a global view, considering the persistent presence, for three months, of cough, fever, arthralgia, an episode of erythema nodosum and the alteration of blood and urine tests. Only after a long period of time a chest $\mathrm{x}$-ray was prescribed and it showed an unclear image of pneumonitis. Chest CT confirmed a granulomatous and interstitial involvement of the lung, and bronchoscopy a hemorrhagic alveolitis. In addition, the renal biopsy showed necrotizing and glomerular vasculitis. This case could have been fatal if untreated, and the patient now needs close follow up to observe stabilization of the disease or new exacerbations.

\section{References}

1. Falk RJ, Gross WL, Guillevin L, et al. Granulomatosis with polyangiitis (Wegener's): an alternative name for Wegener's granulomatosis. J Am Soc Nephrol 2011;22: 587-8.

2. Seo P, Stone JH. The antineutrophil cytoplasmic antibody-associated vasculitides. Am J Med 2004;117:39-50.

3. Mahr AD, Neogi T, Merkel PA. Epidemiology of Wegener's granulomatosis: lessons from descriptive studies and analyses of genetic and environmental risk determinants. Clin Exp Rheumatol 2006;24:S82-91.

4. Miloslavsky E, Specks U, Merkel P, et al. Clinical outcomes of remission induction therapy for severe ANCAassociated vasculitis. Arthritis Rheum 2013;65:2441-9.

5. Pagnoux C, Hogan SL, Chin H, et al. Predictors of treatment resistance and relapse in antineutrophil cytoplasmic antibody-associated small-vessel vasculitis: comparison of two independent cohorts. Arthritis Rheum 2008;58: 2908-18.

6. Mukhtyar C, Guillevin L, Cid MC, et al. EULAR recommendations for the management of primary small and medium vessel vasculitis. Ann Rheum Dis 2009;68:310-7. 